gastroenterologist, underwent investigations and received a GI diagnosis. ROME III criteria were used to categorise FGID. A control group of patients, aged 18-70, who were referred to secondary care for non-GI related problems, were similarly assessed. The prevalence of JHS in various GI diagnoses and in controls, adjusted for age and gender,was compared. Non-GI characteristics and QOL was compared in JHS and non-JHS patients.

Results 688 GI patients [254 organic: (55\% F, 43y); 341 FGID: (65\% F, 40y); 53 reflux: (40\% F, 46y)] and 93 non-GI controls $(67 \% \mathrm{~F}, 43 \mathrm{y})$ were included. JHS prevalence was higher in FGID (38\%) and reflux (40\%) compared to organic disorders $(26 \%)$ and controls $(26 \%)(p=0.003)$. JHS was significantly associated with FGID (ORadj: 1.7, CI:1.02-2.88), specifically postprandial distress syndrome (ORadj 2.2, CI: 1.2-2.2), and with reflux disorders (ORadj 2.2, CI: 1.1-4.7), but not with organic disorders (ORadj: 1.0, CI:0.6-1.8). FGID patients with JHS had significantly more FM (12.6 vs. 4.9\%, p = 0.02), chronic pain (23.2 vs. $11.7 \%, \mathrm{p}=0.01)$, somatisation scores (13 vs. $10, \mathrm{p}<0.01)$, anxiety scores $(0.5$ vs. $0.36, \mathrm{p}=0.02)$ and urinary autonomic symptoms $(30.5 \%$ vs $19.6 \%, \mathrm{p}=0.047)$, and worse pain related QOL scores (45 vs. 63.5, $\mathrm{p}<0.01$ ).

Conclusion JHS is associated with functional dyspepsia, and non-erosive reflux disease, and with FM, chronic pain, somatisation and anxiety. Clinical assessment for JHS in GI clinics is indicated in those with a combination of functional upper GI symptoms and extra-intestinal symptoms as this may help earlier identification of a more 'challenging' group of patients with multiple somatic symptoms and worse QOL. These may benefit from early multidisciplinary approach involving rheumatologists and pain specialists.

Disclosure of Interest None Declared.

\section{PWE-161 THE MACROGOL DRINK TEST TO DISTINGUISH FUNCTIONAL CONSTIPATION (FC) AND CONSTIPATION PREDOMINANT IRRITABLE BOWEL SYNDROME (IBS-C): UNDERLYING MECHANISMS DEMONSTRATED USING MRI}

${ }^{1} \mathrm{C}$ Lam*, ${ }^{2} \mathrm{G}$ Chaddock, ${ }^{2} \mathrm{C}$ Hoad, ${ }^{3} \mathrm{C}$ Costigan, ${ }^{2} \mathrm{E}$ Cox, ${ }^{2} \mathrm{~S}$ Pritchard, ${ }^{4} \mathrm{~K}$ Garsed, ${ }^{2} \mathrm{~L}$ Marciani, ${ }^{2} \mathrm{P}$ Gowland, ${ }^{1} \mathrm{R}$ Spiller. ${ }^{1} \mathrm{NIHR}$ Nottingham Digestive Diseases Biomedical Research Unit, Nottingham, UK; ' ${ }^{2}$ niversity of Nottingham, Nottingham, UK; ${ }^{3}$ Nottingham University Hospital Trusts, Nottingham, UK; ${ }^{4}$ Royal Derby Hospital, Derby, UK

\subsection{6/gutjnl-2014-307263.421}

Introduction Patients with constipation may have either FC or IBS-C which require different treatments. They are often dissatisfied with their treatment because diagnosis relies on symptoms which frequently overlap.
Methods 46 CC patients (24 FC and 22 IBS-C), age 18-68 years, unresponsive to simple laxatives, were compared with 11 healthy volunteers (HV). Whole gut transit (WGT) was assessed using a MRI scan $24 \mathrm{~h}$ following ingestion of 5 marker pills as previously validated. Patients then consumed 1 litre of macrogol (MCG) followed by hourly MRI scans for $4 \mathrm{~h}$ and scored bowel symptoms from 0-10 (none-severe). Colonic movements were assessed using a motility index (MI) based on colonic wall movement and hypersensitivity index (HI) was calculated as bloating symptom/ascending colon (AC) volume.

Results Mean (SD) See Table 1. FC and IBS-C have slower WGT and higher HI than HV. FC showed significantly greater fasting SBWC, AC volume and reduced MI following ingestion of MCG compared to HV and IBS-C. Moreover, FC showed impaired response to MCG with longer time to first bowel movement and reduced stool frequency on the study day when compared with HV and IBS-C. Time to 1 st bowel movement correlated significantly with AC volume $2 \mathrm{~h}$ post MCG, $\mathrm{r}=$ $0.44, p=0.004$ and fasting SBWC, $r=0.34, p=0.035$. Using a cut-off $>230$ min distinguishes FC from IBS-C with sensitivity $55 \%$ and specificity $95 \%$; this needs validation in a repeat study. Conclusion Time to first bowel movement $>230$ min makes IBS unlikely and should help target treatments. Our MI studies show this is dueto greater motility response to distension in IBS-C who has lower fasting SBWC and AC volumes versus FC. IBS-C showed similar features to $\mathrm{HV}$ but can be distinguished by greater $\mathrm{HI}$ following distension which suggest hypersensitivity. This inexpensive test done without MRI could help clinicians to distinguish these 2 conditions.

Disclosure of Interest C. Lam: None Declared, G. Chaddock: None Declared, C. Hoad: None Declared, C. Costigan: None Declared, E. Cox: None Declared, S. Pritchard: None Declared, K. Garsed: None Declared, L. Marciani: None Declared, P. Gowland Consultant for: Merck, Ironwood, Unilever, R. Spiller Grant/research support from: Lessaffre, Ironwood, Consultant for: Almirall, Astellas, Danone and Sanofi, Conflict with: Free drug for clinical trial from Norgine.

\section{PWE-162 AN AUDIT OF TESTING FOR COELIAC DISEASE IN PATIENTS DIAGNOSED WITH THE IRRITABLE BOWEL SYNDROME AT A LARGE PRIMARY CARE CENTRE}

${ }^{1} \mathrm{~J}$ Cooke, ${ }^{2} \mathrm{P}$ Clamp, ${ }^{1} \mathrm{D}$ Haigney, ${ }^{1} \mathrm{~K}$ Rostami, ${ }^{3} \mathrm{D}$ Aldulaimi*. ${ }^{1}$ Institute of Health and Social Care, Univeristy of Worcester, Worcester, UK; ${ }^{2}$ Primary Care, St Johns Surgery, Bromsgrove, UK; ${ }^{3}$ General Medicine, Worcestershire Acute Hospitals NHS Trust, Worcester, UK

\subsection{6/gutjpl-2014-307263.422}

Introduction NICE guidance recommends that patients presenting with symptoms suggestive of the irritable bowel syndrome

\begin{tabular}{|c|c|c|c|c|}
\hline Mean (SD) & HV $(n=11)$ & $\mathrm{FC}(\mathrm{n}=23)$ & IBS-C $(n=20)$ & $P$ value \\
\hline WGT (h) & 30.4 & $108.2^{*}, * *$ & $71.4^{*}$ & $<0.0001$ \\
\hline Fasting small bowel water content (SBWC) (ml) & $83(64)$ & $114(97)^{* \star}$ & $57(61)$ & 0.0383 \\
\hline Fasting AC (ml) & $193(84)$ & $314(100)^{*, * *}$ & $219(66)$ & 0.0002 \\
\hline AC volume $2 \mathrm{~h}$ post ingestion of MCG (ml) & $357(153)$ & $597(170)$ & $376(163)$ & $<0.0001$ \\
\hline MI $2 \mathrm{~h}$ post ingestion of MCG (s) & $80.2(48.1)$ & $28.3(35.1)^{*, \star \star}$ & $56.4(42.9)$ & 0.0044 \\
\hline Time to first bowel movement (min) & $117.3(62.4)$ & $588(1034)^{* * * *}$ & $97.3(72)$ & 0.0001 \\
\hline Bowel frequency on study day & $7.8(2.7)$ & $3.9(4.1)^{*, * \star}$ & $7.8(3.0)$ & $<0.0001$ \\
\hline Hypersensitivy Index $\left(I^{-1}\right)$ & $5.7(4.9)$ & $12.3(6.6)^{*}$ & $16.6(14)^{*}$ & 0.0133 \\
\hline \multicolumn{5}{|c|}{${ }^{*} \mathrm{p}<0.05$ compared to $\mathrm{HV},{ }^{* *} \mathrm{p}<0.05$ compared to IBS-C. } \\
\hline
\end{tabular}


(IBS) should be tested for coeliac disease (CD). This study reviewed the symptoms, demographics and investigation of pateints diagnosed with IBS at a large semi-rural general practice.

Methods Patients diagnosed with the IBS were identified from a computer database of 11,250 patients at St Johns Surgery. 250 casenotes were randomly selected for analysis. Patient demographics, symptoms recorded and investigations performed were recorded. a history of depression was recorded. Census and postcode data was used as a surogate marker of poverty.

Results 499 patients had a diagnosis of IBS (110 male and 389 females). The records of 48 male and 202 female patients were reviewed.

186 patients were diagnosed with IBS within 6 months of their initial consultation. 180 began treatment for IBS within 6 months of diagnosis.

65 patients were tested for CD (13 males and 52 females). 34 $(52 \%)$ of those tested for CD went on to have further investigations such as a barium enema or colonoscopy.185 patients were not tested for CD. 137 had no further investigations.

Symptoms of those tested for CD were a change in bowel habit (90.8\%), abdominal pain (90.8\%), chronic diarrhoea $(73.8 \%)$ and bloating (67.7\%). Symptoms of those not tested for $\mathrm{CD}$ were the most common symptoms where abdominal pain (91.9\%), change in bowel habit (73.5\%) and bloating (69.2\%) and chronic diarrhoea (52.4\%).

There was no significant difference in poverty or history of depression between those tested and not tested for CD.

Conclusion This study suggests that patients diagnosed with the IBS in primary care are not routinely tested for CD. Explanations include a pragmatic approach to investigation of common gastrointestinal symptoms and a likely difference between patients diagnosed with the IBS in primary care and those referred onto secondary care and academic centres.

\section{REFERENCES}

National Institute for Health and Clinical Excellence. 2009. Recognition and Assessment of Coeliac Disease

National Institute for Health and Clinical Excellence. 2008. Irritable bowel syndrome in adults

Disclosure of Interest None Declared.

\section{PWE-163 THE EXCITATORY EFFECTS OF REPETITIVE CEREBELLAR BRAIN STIMULATION ON HUMAN SWALLOWING MOTOR PATHWAYS ARE CRITICALLY DEPENDENT ON STIMULUS DURATION}

DH Vasant*, E Michou, S Mistry, S Hamdy. Gastrointestinal Centre, The University of Manchester, Salford Royal NHS Foundation Trust, UK

\subsection{6/gutjnl-2014-307263.423}

Introduction Previously we have demonstrated frequency-specific effects of cerebellar repetitive transcranial magnetic stimulation (rTMS) in enhancing human cortical swallowing pathways, ${ }^{1}$ as a prelude to developing therapeutic parameters for post-stroke dysphagia. Here, we investigate the durational parameters of $10 \mathrm{~Hz}$ cerebellar rTMS on pharyngeal motor excitability.

Methods Healthy subjects $(\mathrm{n}=12,7$ male, 5 female, mean age $31 \pm 4$ years), were intubated with an intraluminal catheter to record pharyngeal electromyography before bilateral MRI-guided single-pulse TMS mapping of pharyngeal motor evoked potentials (PMEPs) to co-localise optimal cortical and cerebellar sites for pharyngeal activity. Baseline cortical PMEPs were then

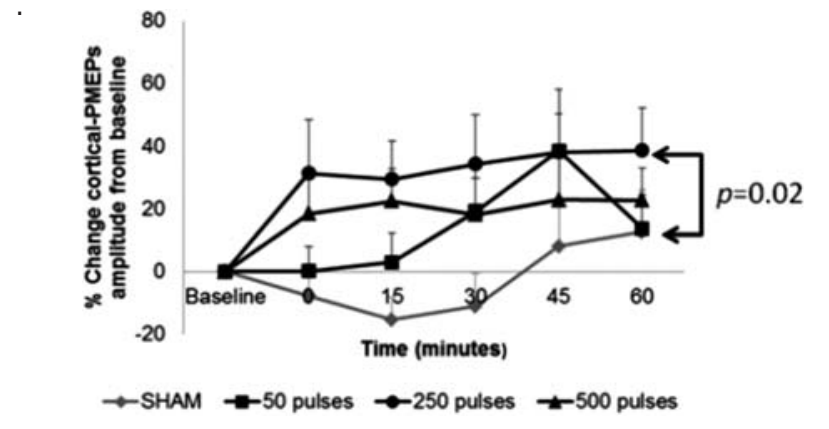

Abstract PWE-163 Figure 1

recorded followed by one of the four cerebellar interventions; sham (coil tilted to $90^{\circ}$ ), short duration (50-pulses), intermediate duration (250-pulses) or longer-train (500-pulses) at $10 \mathrm{~Hz}$ frequency, received on separate randomised visits. Post-intervention PMEPs were recorded for up to an hour as a measure of cortical excitability. Normalised (percentage change from baseline) PMEP data were then compared to sham using repeated measures ANOVA (factors of time, hemispheric site, intervention).

Results Intermediate train-length (250-pulses: $\mathrm{F}_{1,11}=7.3, p=$ 0.02 ) was most effective at increasing pharyngeal cortical excitability bilaterally compared to longer (500-pulses: $\mathrm{F}_{1,11}=4.5, \mathrm{p}$ $=0.058)$ and shorter (50 pulses; $\left.\mathrm{F}_{1,11}=1.7, \mathrm{p}=0.21\right) 10-\mathrm{Hz}$ cerebellar interventions (Figure 1).

Conclusion Our data confirm that sustained facilitation of the pharyngeal motor cortex to cerebellar rTMS is not only dependent on the stimulation frequency, but also the duration of stimulation. We therefore propose that 250-pulses of $10 \mathrm{~Hz}$ cerebellar rTMS to be the optimal parameters for future therapeutic studies in post-stroke dysphagia.

\section{REFERENCE}

1 Vasant $\mathrm{DH}$, et al. Neuronavigated repetitive cerebellar stimulation produces longlasting activation of human cortical swallowing projections. Gut 2013;62:A16A17

Disclosure of Interest

None Declared.

\section{PWE-164 A META-ANALYSIS OF RANDOMISED CONTROLLED TRIALS ON THE EFFECT OF PROBIOTICS ON FUNCTIONAL CONSTIPATION IN ADULTS}

${ }^{1,2}{ }^{E}$ Dimidi ${ }^{*},{ }^{1,2} \mathrm{~S}$ Christodoulides, ${ }^{3} \mathrm{KC}$ Fragkos, ${ }^{2} \mathrm{SM}$ Scott, ${ }^{1} \mathrm{~K}$ Whelan. ${ }^{1}$ Diabetes and Nutritional Sciences Division, King's College London, UK; ${ }^{2}$ Neurogastroenterology Group, Queen Mary University of London, UK; ${ }^{3}$ Centre for Gastroenterology and Clinical Nutrition, University College London, London, UK

\subsection{6/gutjnl-2014-307263.424}

Introduction Constipation is a prevalent gastrointestinal disorder whose treatment remains challenging. Patient dissatisfaction with prescribed medications is common, and there is a need for alternative methods of management. Probiotics are live microorganisms that, when administered in adequate amounts, confer a health benefit to the host, and have been increasingly used in the management of functional constipation. The aim of this study was to determine the effect of probiotics in functional constipation through a systematic review and meta-analysis of randomised controlled trials (RCTs) in adults.

Methods Methods followed PRISMA recommendations. Studies were identified by searching four electronic databases, backsearching reference lists, contacting authors and hand searching 